\title{
Optimization of Lipid-Lowering Therapy for High Cardiovascular Risk Patients Through Electronic Medical Record Reporting and Pharmacist Evaluation
}

Kaylee Chen, PharmD; Scott Canfield, PharmD, CSP; Joshua Blackwell, PharmD, MS; Samuel Houmes, PharmD, BCACP; Steven Jones, MD; and John Lindsley, PharmD, BCPS-AQ Cardiology

\begin{abstract}
BACKGROUND: Identification of high cardiovascular risk patients on suboptimal lipid-lowering therapy (LLT) may be possible through electronic medical record (EMR) reporting, presenting an opportunity for pharmacist involvement in optimizing drug regimens.

OBJECTIVES: To (a) identify high cardiovascular risk patients with opportunities for LLT optimization through EMR reporting and (b) evaluate effectiveness of pharmacist review and treatment algorithm on recommending treatment modifications compared with algorithm application alone.
\end{abstract}

METHODS: We generated an EMR report to identify adult patients aged 21-75 years with clinical atherosclerotic cardiovascular disease and low-density lipoprotein cholesterol (LDL-C) level $\geq 70 \mathrm{mg} / \mathrm{dL}$ during a 6 -month period and collected pertinent data elements. We selected a subgroup of patients for remote pharmacist review and determined recommendations based on our predefined LLT optimization algorithm and pharmacist clinical judgment. One pharmacist was responsible for making all recommendations and communicated potential treatment modification to primary care providers via email and/or EMR messaging. We tracked provider acceptable/rejection rate to all recommendations made. We also compared recommendations based on using the algorithm alone to combining pharmacist chart review and algorithm and examined reasons for any discrepancies.

RESULTS: 941 patients met inclusion criteria, with 399 patients (42.4\%) not currently on any LLT. At baseline, 249 patients (25.3\%) were on a highintensity statin, and $19(1.9 \%)$ were on a proprotein convertase subtilisin/ kexin type 9 inhibitor. A subgroup of 34 patients were reviewed, of which $30(88.2 \%)$ were on suboptimal therapy despite not achieving LDL-C goals. The pharmacist recommended to intensify statin therapy for 16 patients (47.1\%), initiate nonstatin therapy for 9 patients $(26.5 \%)$, and initiate statin therapy in 5 patients $(14.7 \%)$. Pharmacist recommendation acceptance rate was $53.3 \%$, with no response received in $26.6 \%$ of cases. The algorithm evaluation alone yielded the same recommendation as the combined pharmacist review with algorithm in $30(88.2 \%)$ of the cases and differed in 4 cases.

CONCLUSIONS: The underutilization of LLT among high cardiovascular risk patients remains a growing issue despite effective treatment options with cardiovascular benefits. Pharmacists may be able to identify these patients by using reportable EMR data elements and applying a treatment optimization algorithm to make therapy recommendations and improve outcomes.

J Manag Care Spec Pharm. 2020;26(8):1010-16

Copyright $\odot 2020$, Academy of Managed Care Pharmacy. All rights reserved.

\section{What is already known about this subject}

Lipid-lowering therapy among high cardiovascular risk patients is underused.

Pharmacists are well positioned to review clinical data and provide recommendations for lipid therapy optimization.

\section{What this study adds}

Identification of high-risk patients with gaps in lipid-lowering therapy may be possible with electronic medical record reporting. Use of a predefined treatment algorithm yielded similar recommendations as pharmacist chart review in lipid therapy optimization for high-risk patients.

H yperlipidemia is a well-established risk factor for developing atherosclerotic cardiovascular disease (ASCVD), the leading cause of death in the United States. ${ }^{1}$ Despite current practice guidelines advocating statin and select nonstatin use, underuse of hyperlipidemia therapy has been observed among high-risk populations. Of 17 million American adults living with ASCVD, 63\% are found to be on statin therapy, of which $80 \%$ are not at low-density lipoprotein cholesterol (LDL-C) goal. ${ }^{2,3}$

The 2018 American College of Cardiology and American Heart Association (ACC/AHA) Guideline on Management of Blood Cholesterol provided several updates to the 2013 ACC/AHA guidelines for secondary ASCVD prevention in high-risk patients. ${ }^{4,5}$ First, an LDL-C goal of $<70 \mathrm{mg} / \mathrm{dL}$ is recommended for secondary prevention in these high-risk patients. Nonstatin medications with positive cardiovascular benefits, namely ezetimibe and the proprotein convertase subtilisin/kexin type 9 (PCSK9) inhibitors evolocumab and alirocumab, are recommended for patients with high ASCVD risk requiring additional LDL-C reduction if unable to achieve the goal of $70 \mathrm{mg} / \mathrm{dL}$ after a maximally tolerated statin in a sequential approach. If LDL-C levels remain $\geq 70 \mathrm{mg} / \mathrm{dL}$ on maximal lipid-lowering therapy (LLT), then adding a PCSK9 inhibitor is reasonable. 
Key elements such as diagnosis, LDL-C, and current drug therapy are required to make treatment decisions for patients. Since these elements are commonly documented as discrete data within the electronic medical record (EMR), an opportunity may exist to leverage data in the identification of high-risk patients with LLT regimens not currently optimized. This study aims to identify high cardiovascular risk patients with opportunities for LLT optimization through the generation of an EMR report and to evaluate effectiveness of a pharmacist chart review in addition to treatment optimization algorithm application on recommending treatment modifications compared with algorithm application alone.

\section{Methods}

Johns Hopkins Medicine includes 6 academic and community hospitals, 4 suburban health care and surgery centers, and over 40 patient care locations, including home and community care. This study evaluated patients who received care in any Johns Hopkins ambulatory patient care clinic or medical campus in the state of Maryland and in Washington, DC. The first phase of this study involved identification and characterization of patients with clinical ASCVD requiring secondary prevention and not at LDL-C goal, based on development of an EMR report. The second phase of the study included determination of the appropriateness of current LLT regimens and opportunities for treatment optimization for a subgroup of patients through a pharmacist review of patient charts and the use of a treatment optimization algorithm.

We generated an EMR report to identify patients who met inclusion criteria between March 30, 2018, and September 30, 2018. We chose to collect data during this 6-month period in order to capture only patients with recently collected LDL-C levels that could reasonably be used by clinicians to make real-time modification recommendations. Based on the 2018 ACC/AHA guidelines, adults aged 21-75 years with a clinical ASCVD diagnosis and LDL-C level $\geq 70 \mathrm{mg} / \mathrm{dL}$ were included, while patients who were pregnant or without a documented LDL-C level within the study period were excluded. We defined clinical ASCVD diagnosis as the presence of an International Classification of Diseases, Tenth Revision, Clinical Modification code for acute coronary syndromes, history of myocardial infarction, stable or unstable angina, coronary or other arterial revascularization, stroke, transient ischemic attack, or peripheral vascular conditions presumed to be of atherosclerotic origin within the patient problem list during the specified time frame.

We also collected baseline patient characteristics and laboratory values, as well as each patient's primary care provider (PCP), documented statin intolerance, current hyperlipidemia therapy, and current medications known to have major drug interactions with statins. We identified statin intolerance through the patient allergy section. Interacting medications with statins collected included cyclosporine, fibrates, bile acid binding resins, verapamil, diltiazem, colchicine, clarithromycin, itraconazole, and protease inhibitors. PCPs consisted of physicians and certified registered nurse practitioners specialized in internal medicine or family practice.

We developed a treatment optimization algorithm based on the 2018 ACC/AHA guidelines to facilitate determination of treatment modification (Figure 1). The algorithm served to identify appropriate treatment recommendations based on data elements collected from the EMR. We selected a subgroup of patients for pharmacist chart review and algorithm evaluation by determining 15 local PCPs with the highest number of patients meeting inclusion criteria and contacted those prescribers via email to gauge their willingness to participate in the project. For subgroup analysis, we included only patients of the providers who were willing to participate. The pharmacist who performed chart reviews was responsible for making all recommendations and had no established relationships with participating PCPs. The pharmacist reviewed charts remotely and was not based within provider practices.

We categorized recommendations made by following the optimization algorithm, as well as recommendations made after additional pharmacist review into 1 of 4 types: (1) addition of statin therapy, (2) intensification of statin therapy, (3) addition of nonstatin therapy, and (4) no modification to therapy. Using the ACC/AHA guidelines, we classified the intensity of statin therapy into 3 groups: high intensity, moderate intensity, and low intensity. As part of prescriber subgroup inclusion, the pharmacist determined prescriber communication preferences before making recommendations and ultimately communicated treatment addition or modification recommendations through email and/or EMR messaging for all included prescribers. We defined "no response" as no answer from the PCP after 3 follow-up outreaches. The pharmacist also referred patients deemed to be at the highest risk to a lipid specialist.

Following pharmacist review, we descriptively compared the recommendation results from using the optimization algorithm alone with a combination of pharmacist chart review and algorithm evaluation. The pharmacist identified the number of differences in recommendations from the developed algorithm compared with chart review and examined reasons for the discrepancies. We further categorized the identified reasons for discrepancies between recommendations made by using the different methods. Reasons for recommendation differences identified included EMR reporting limitations, as well as limitations as a result of using the algorithm alone. 


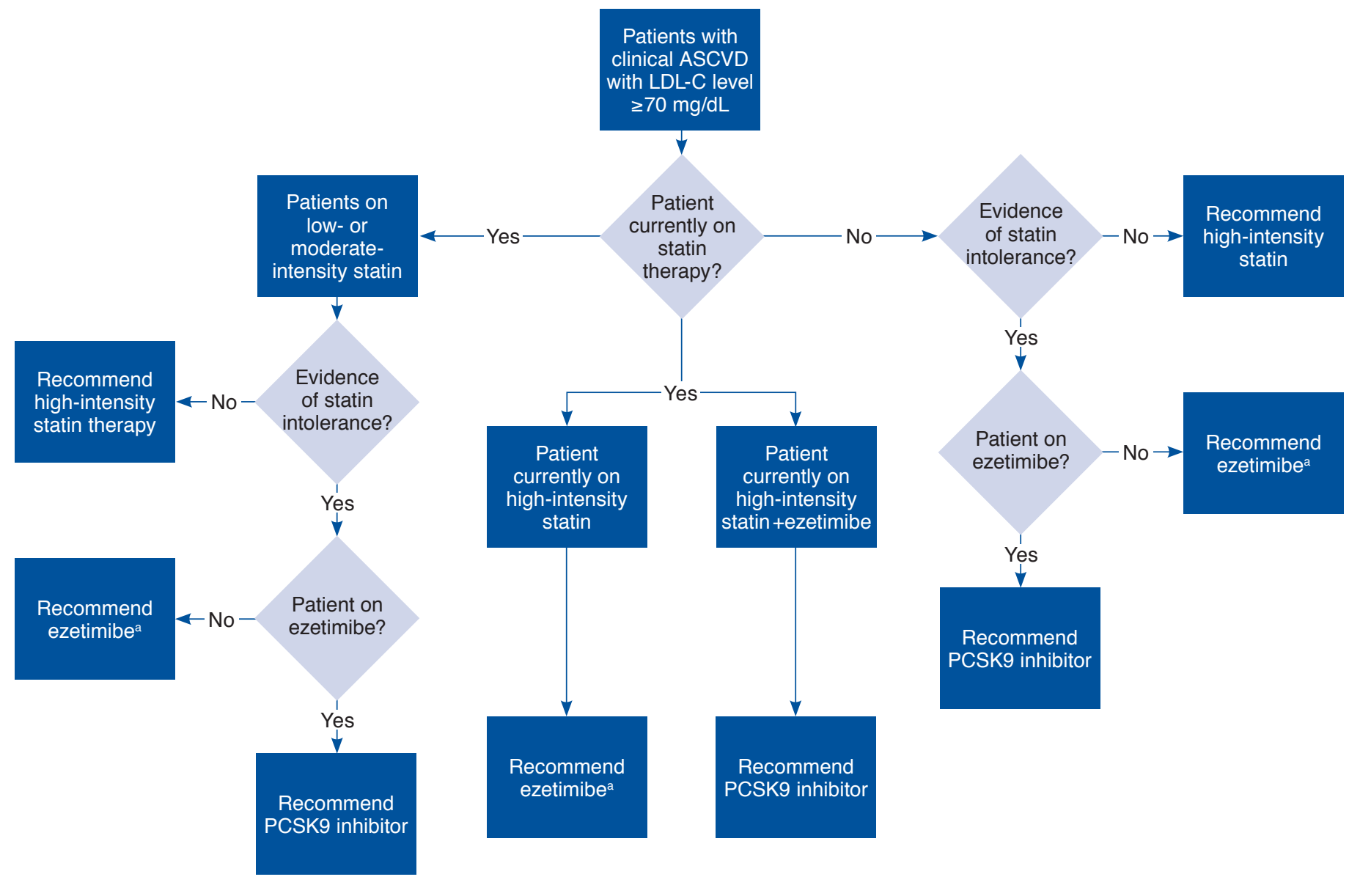

a If goal LDL-C $<70 \mathrm{mg} / \mathrm{dL}$ is not achieved with addition of ezetimibe or intensification of statin therapy, a PCSK9 inhibitor should be considered. $A S C V D=$ atherosclerotic cardiovascular disease; $L D L-C=l o w$-density lipoprotein cholesterol; $P C S K 9=$ proprotein convertase subtilisin/kexin type 9.

This quality improvement research project did not warrant submission to the Institutional Review Board (IRB) per the IRB review decision tool algorithm developed under a joint agreement between the IRB and Department of Pharmacy at the institution. All work conducted for the research occurred with the approval from the Center for Pharmaceutical Outcomes and Policy. No statistical comparisons were performed in this study; all data were presented descriptively.

\section{Results}

A total of 941 patients met inclusion requirements (Table 1). Average age of the study population was 63 years, with mean LDL-C level of $105 \mathrm{mg} / \mathrm{dL}$ and mean calculated body mass index (BMI) of $30.5 \mathrm{~kg} / \mathrm{m}^{2}$. While 542 patients (57.6\%) received at least 1 LLT, 44 patients (4.7\%) were on more than
1 medication. Two hundred forty-nine patients (25.3\%) were on a high-intensity statin; 62 patients (6.3\%) were on ezetimibe; and 19 (1.9\%) patients were on a PCSK9 inhibitor at baseline. Of the patients on a PCSK9 inhibitor, 2 (10.5\%) also received a high-intensity statin; 3 (15.8\%) were on a high-intensity statin plus ezetimibe; 5 (26.3\%) were on ezetimibe; and 9 (47.4\%) not on any other hyperlipidemia medications.

The subgroup analysis included 34 patients, who were reviewed by the pharmacist. Mean LDL-C for this group was $100 \mathrm{mg} / \mathrm{dL}$. Of these patients, 30 (88.2\%) received suboptimal LLT regimens despite LDL-C levels not at goal. The pharmacist made a recommendation to intensify statin therapy for 16 patients (47.1\%), initiate nonstatin therapy for 9 patients (26.5\%), and initiate statin therapy for 5 patients (14.7\%). Four patients (11.7\%) did not require any changes to therapy; 


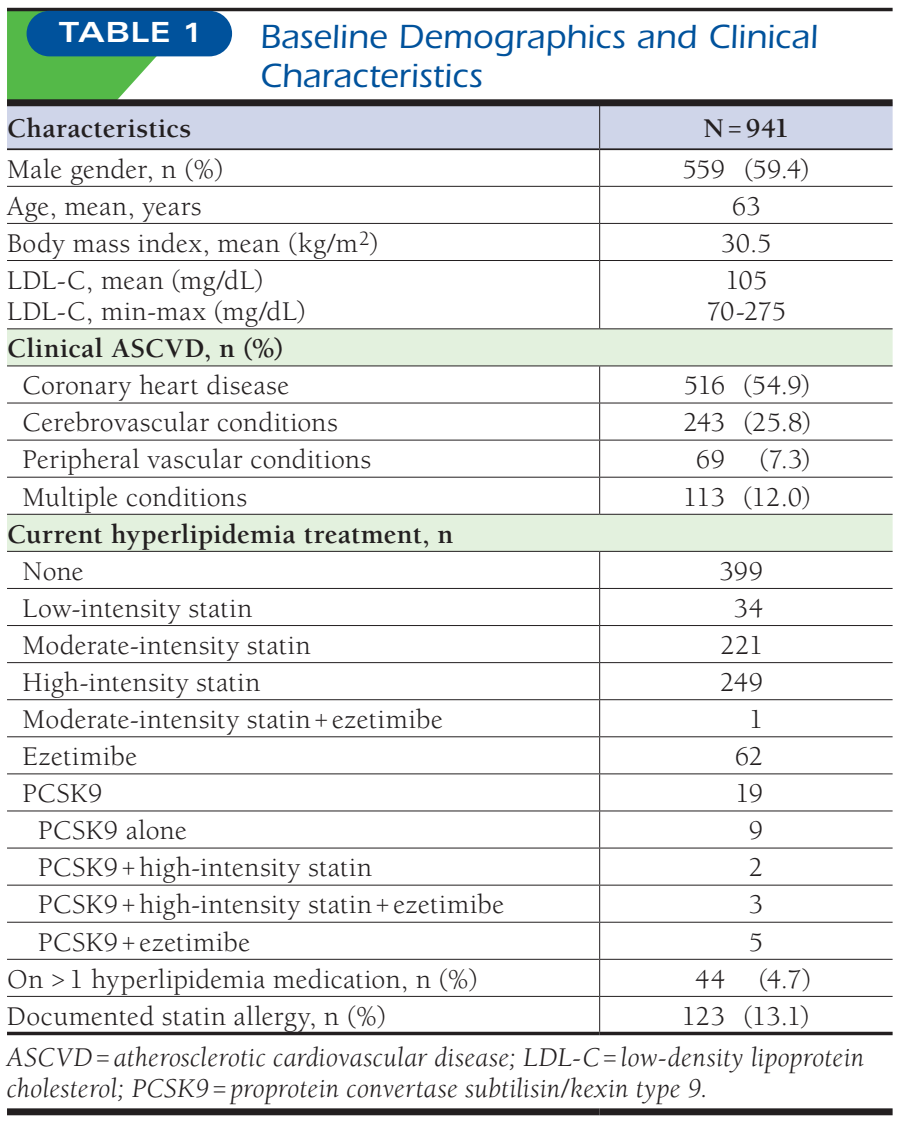

3 patients had incorrect diagnoses entered in their charts; and 1 patient had a repeat LDL-C level outside of data collection parameters at goal. Of the 30 patients with treatment modification opportunities, providers accepted 16 (53.3\%) pharmacist recommendations, rejected $6(20.0 \%)$ recommendations, and did not provide responses for 8 (26.6\%) patients. Providers did not accept recommendations in 6 cases because of more urgent issues to address for 2 patients and 1 occurrence of each for the following reasons: patient resistance to medication changes, lack of adherence to current hyperlipidemia regimen, myocardial infarction not of atherosclerotic origin, and repeat LDL-C level required.

The algorithm and pharmacist review yielded the same recommendations in 30 (88.2\%) of the cases and differed in 4 cases. In 2 of the cases, the report could not capture statin intolerances not listed in the patient allergy section; as a result, the pharmacist recommended adding ezetimibe over increasing the statin dose because of evidence of muscle pain/weakness after chart review. Of note, the EMR report also did not differentiate between the statins to which patients had intolerances. As a result, despite the algorithm's recommendation to add ezetimibe in 1 case, the pharmacist recommended increasing the rosuvastatin dose instead, since the patient's intolerance was to atorvastatin. The algorithm and pharmacist review recommendations differed for 1 of the patients who required no therapy modifications. Since the repeat LDL-C outside of the data reporting time frame was $<70 \mathrm{mg} / \mathrm{dL}$, the pharmacist recommended no changes despite the algorithm's initial recommendation to increase statin intensity. Of the 4 varying recommendations, 2 were accepted (50\%); 1 rejected (25\%); and 1 received no response (25\%).

Of the 34 subgroup analysis patients, the pharmacist recommended the initiation of a PCSK9 inhibitor for 3 (8.8\%) patients. Given the estimated reduction of LDL-C with dose increases in statin therapy and addition of ezetimibe, a PCSK9 inhibitor may be beneficial in $8(23.5 \%)$ of the patients to achieve LDL-C goal. Of note, none of the patients reviewed warranted a referral to a specialist per the pharmacist's judgement.

\section{Discussion}

LLT among high cardiovascular risk patients was underused in our patient cohort. Over a 6-month time period, 941 patients with clinical ASCVD had suboptimal hyperlipidemia therapy despite not achieving LDL-C goal. About $42.4 \%$ of these patients were not receiving any LLT recommended by ACC/ AHA guidelines, and only $4.7 \%$ of patients were taking more than 1 hyperlipidemia medication. Although not assessed with this study, lifestyle intervention plays a vital role in hyperlipidemia management. With this patient population, an opportunity existed for lifestyle interventions (e.g., weight loss) as part of management, evidenced by the number of patients with an overweight or obese BMI classification. Of the 789 patients with a documented height and weight, 293 (37.1\%) had a BMI $\geq 25 \mathrm{~kg} / \mathrm{m}^{2}$ (overweight), and 362 (45.9\%) had a $\mathrm{BMI} \geq 30 \mathrm{~kg} / \mathrm{m}^{2}$ (obese). Further classification showed 189 (24.0\%) patients in class I obesity with BMIs of 30 to $<35 \mathrm{~kg} / \mathrm{m}^{2}$, $114(14.4 \%)$ in class II with BMIs of 35 to $<40 \mathrm{~kg} / \mathrm{m}^{2}$, and 59 (7.4\%) in class III with BMIs $\geq 40 \mathrm{~kg} / \mathrm{m}^{2}$. The mean BMI was $30.5 \mathrm{~kg} / \mathrm{m}^{2}$

Not only was LLT underutilization seen in the overall patient population, we also observed this trend in the subgroup patient analysis. In this subgroup, $88.2 \%$ of the patients reviewed by the pharmacist did not have optimal therapy despite not achieving LDL-C goal. Previous studies have demonstrated that pharmacists are well positioned to obtain and review these data and provide recommendations for therapy optimization leading to patients being more likely to attain target LDL-C compared with those receiving standard of care services without pharmacist involvement. In our study, the pharmacist determined that the majority of patients where a recommendation for change was warranted would benefit 
from modifications of statin therapy. This finding suggests that further evaluation of appropriate statin use and dosing may be required among primary care providers to ensure that LLT is routinely being reassessed for high-risk patients needing secondary prevention of ASCVD.

Previous studies have shown suboptimal LLT use among high cardiovascular risk patient populations. Estimates of statin use for secondary prevention have varied between 50\% and 68\%. ${ }^{6}$ Karalis et al. (2018) evaluated treatment patterns in PCSK9 inhibitor-eligible patients identified through EMR data and found that $<0.5 \%$ of 333,669 patients with ASCVD were prescribed a PCSK9 inhibitor. ${ }^{7}$ Of these patients, only 34\% received high-intensity statins at baseline, and 4\% were prescribed ezetimibe. ${ }^{7}$ Cannon et al. (2017) analyzed medical and pharmacy claims to identify patients with ASCVD and estimate the percentage of patients who would require a PCSK9 inhibitor when oral LLT is maximally intensified. ${ }^{8} \mathrm{~A}$ cohort of 105,269 patients were entered into a simulation, which applied stepwise LLT intensification algorithms to those with LDL-C $\geq 70 \mathrm{mg} / \mathrm{dL}{ }^{8}$ Before treatment intensification, $53.2 \%$ received statins alone or in combination with ezetimibe at baseline, with only $25.2 \%$ having achieved LDL-C goal $<70 \mathrm{mg} / \mathrm{dL} .{ }^{8}$ After simulation of treatment intensification, $99.3 \%$ could achieve LDL-C goal, with $86 \%$ on statins alone or in combination with ezetimibe and 14\% requiring an add-on PCSK9 inhibitor. ${ }^{8}$

In the subgroup analysis of our study, we identified that a PCSK9 inhibitor would likely be beneficial and appropriate in $23.5 \%$ of patients with elevated LDL-C levels. Although patients at our institution were historically likely to see a specialist before receiving a PCSK9 inhibitor, these therapies may be increasingly prescribed by primary care providers because of the high prevalence of uncontrolled hyperlipidemia, relaxing insurance plan prior authorization requirements, as well as changes in the price of these medications. Over the past year, the prices of evolocumab and alirocumab have been reduced by approximately $60 \%$ since coming to market. ${ }^{9}$ Education for prescribers and patients is crucial to ensure that the benefits of these medications and other LLT are known and to increase willingness to use them. Pharmacists are well positioned to support prescribers and patients in increasing awareness of these therapies, as well as encouraging their appropriate use.

Pharmacist recommendation acceptance rate in our study was low (53.3\%), although similar to those reported in other published studies. Boesen et al. (2011) found that prescriber approval rate for pharmacist recommendations communicated by fax in medication therapy management programs (MTMP) was $47.2 \%{ }^{10}$ In another study of MTMP, Michaels et al. (2010) observed that the prescriber acceptance rate of pharmacist recommendations ranged from $42.0 \%$ to $59.8 \% .{ }^{11}$ In our study, most providers were hesitant to make therapy changes immediately, providing reasons such as patients not having follow-up appointments scheduled within the coming weeks or needing repeat LDL-C levels before changes, despite the EMR report's effectiveness in identifying opportunities for LLT optimization for the high-risk patients. The response rate may have been improved with use of other means of communication (e.g., phone or in person) or if the pharmacist had previously established relationship with the providers. Increased education regarding guideline updates and reminders for providers to reevaluate LLT routinely after lab results may help improve treatment optimization and minimize delays in getting patients on optimal therapy.

The algorithm evaluation alone yielded the same recommendation as the combined pharmacist review with algorithm evaluation in 30 (88.2\%) of the cases and differed in 4 cases. Of the 4 varying recommendations, we observed a provider acceptance rate similar to our overall acceptance rate. The reasons for discrepancies in recommendations were largely because of limitations of the report generation and may be minimized with modifications in how certain data elements were pulled. For example, by identifying the specific statin agent documented to which the patient had an intolerance would have provided some clarification in the appropriate next step. Pharmacist chart review of the 34 patients took approximately 355 minutes, averaging about 10 minutes per patient. The low number of discrepancies observed in our study may not provide enough justification for using a pharmacist's time for chart review compared with applying the algorithm alone. Refinement of algorithm design and report generation could further decrease the number of discrepancies seen with algorithm recommendations alone compared with the addition of the pharmacist review.

Some strategies to improve the utility of the algorithm and EMR reporting achieving LDL-C goals may include flagging of high-risk patients based on data elements commonly available within the EMR or developing other clinical decision support systems. Tools such as best practice alerts in the EMR could serve as a method to prompt providers to reassess LLT upon elevated LDL-C results for patients with clinical ASCVD. Studies have shown that clinical decision support systems can increase provider adherence to clinical guidelines through methods such as standardized order sets, alerts to specific protocols, and assistance with managing patients on treatment protocols. ${ }^{12}$ It is important to be mindful of potential pitfalls associated with these tools, such as alert fatigue, and careful consideration should be given to the exploration of automating the identification of opportunities for LLT therapy optimization. 


\section{Limitations}

Our study had several limitations. The retrospective design and small subgroup of patients reviewed limited the ability to draw firm conclusions regarding the best method to prompt LLT optimization. We did not aim to identify and characterize patients with ASCVD who had reached an LDL-C $<70 \mathrm{mg} / \mathrm{dL}$ or those who did not have LDL-C levels documented during our study time frame and, thus, may have missed patients who may have warranted additional laboratory monitoring or may have had LDL-C levels $>70 \mathrm{mg} / \mathrm{dL}$ documented less frequently or were simply unmonitored.

The subgroup of patients was not a randomized selection, which could have introduced bias based on participating providers. Adherence and lifestyle interventions were unable to be accurately assessed for the purposes of this study and were left to be addressed by providers. Accurate assessment of adherence and lifestyle interventions would have helped further determine whether additional therapy was the appropriate next step.

The effectiveness of the algorithm was limited by available data and accuracy of documentation within the EMR. Finally, patients were drawn from clinical practice at an academic medical system and may not be generalizable to patients seen in other settings.

\section{Conclusions}

$\overline{\text { Despite the availability and proven effectiveness of statin and }}$ select nonstatin therapies, LLT among high cardiovascular risk patient populations continues to be suboptimal and underused as demonstrated by this study. The study highlights the need to provide education and support in primary care settings in order to increase awareness and willingness to use appropriate LLT. Findings suggest that strategies to improve the achievement of LDL-C goals among high-risk patients are necessary and could include pharmacist evaluation of high-risk patient populations and/or the flagging of high-risk patients for LLT re-evaluation based on data elements commonly available within the EMR. High-risk criteria defined in this study may serve as a foundation to consider for future studies, but additional research should be undertaken to determine the most appropriate method of alerting providers to reassess high-risk patients and their LLT regimens.

\section{Authors}

KAYLEE CHEN, PharmD; SCOTT CANFIELD, PharmD, CSP; JOSHUA BLACKWELL, PharmD, MS; and SAMUEL HOUMES, PharmD, BCACP, Johns Hopkins Home Care Group, Baltimore, Maryland. STEVEN JONES, MD, Johns Hopkins University School of Medicine, Baltimore, Maryland, and JOHN LINDSLEY, PharmD, BCPS-AQ Cardiology, The Johns Hopkins Hospital, Baltimore, Maryland.

AUTHOR CORRESPONDENCE: Kaylee Chen, PharmD, Specialty Pharmacist Navigator, Reading Hospital Ambulatory Pharmacy, 420 S. 5th Ave., Reading, PA 19611. Email: 1kaylee.chen@gmail.com.

\section{DISCLOSURES}

This research did not receive any specific grant from funding agencies in the public, commercial, or not-for-profit sectors. The authors have no relevant declarations of interest to disclose.

This study was presented as a poster presentation at the APhA Annual Meeting, March 2019, Seattle, WA, and as a platform presentation at the Eastern States Conference, May 2019, Hershey, PA.

\section{REFERENCES}

1. Centers for Disease Control and Prevention. Leading causes of death March 2017. Available at: https://www.cdc.gov/nchs/fastats/leading-causesof-death.htm. Accessed June 8, 2020.

2. Wong ND, Young D, Zhao Y, et al. Prevalence of the American College of Cardiology/American Heart Association statin eligibility groups, statin use, and low-density lipoprotein cholesterol control in U.S. adults using the National Health and Nutrition Examination Survey 2011-2012. J Clin Lipidol. 2016;10(5):1109-18.

3. Centers for Disease Control and Prevention. Cholesterol fact sheet. Available at: https://www.cdc.gov/dhdsp/data_statistics/fact_sheets/fs_cholesterol.htm. Accessed June 8, 2020

4. Stone NJ, Robinson JG, Lichtenstein AH, et al.; American College of Cardiology/American Heart Association Task Force on Practice Guidelines. 2013 ACC/AHA guideline on the treatment of blood cholesterol to reduce atherosclerotic cardiovascular risk in adults: a report of the American College of Cardiology/American Heart Association Task Force on Practice Guidelines. J Am Coll Cardiol. 2014;63(25 Pt B):2889-934.

5. Grundy SM, Stone NJ, Bailey AL, et al. 2018 AHA/ACC/AACVPR/ AAPA/ABC/ACPM/ADA/AGS/APhA/ASPC/NLA/PCNA Guideline on the Management of Blood Cholesterol: a report of the American College of Cardiology/American Heart Association Task Force on Clinical Practice Guidelines. J Am Coll Cardiol. 2019;73(24):e285-e350.

6. Johansen ME, Green LA, Sen A, Kircher S, Richardson CR. Cardiovascular risk and statin use in the United States. Ann Fam Med. 2014;12(3):215-23.

7. Karalis DG, Mallya UG, Ghannam AF, et al. Prescribing patterns of proprotein convertase subtilisin-kexin type 9 inhibitors in eligible patients with clinical atherosclerotic cardiovascular disease or heterozygous familial hypercholesterolemia. Am J Cardiol. 2018;121(10):1155-61.

8. Cannon CP, Khan I, Klimchak AC, et al. Simulation of lipid-lowering therapy intensification in a population with atherosclerotic cardiovascular disease. JAMA Cardiol. 2017;2(9):959-66. 
9. Pagliarulo N. Regeneron, Sanofi cut PCSK9 list price, matching earlier move by rival Amgen. BioPharma Dive. February 11, 2019. Available at: https://www.biopharmadive.com/news/regeneron-sanofi-cut-pcsk9-listprice-matching-earlier-move-by-rival-amge/548147/. Accessed June 8, 2020.

10. Boesen KP, Perera PN, Guy MC, Sweaney AM. Evaluation of prescriber responses to pharmacist recommendations communicated by fax in a medication therapy management program (MTMP). J Manag Care Pharm. 2011;17(5):345-54. Available at: https://www.jmcp.org/doi/10.18553/ jmcp.2011.17.5.345.
11. Michaels NM, Jenkins GF, Pruss DL, Heidrick JE, Ferreri SP. Retrospective analysis of community pharmacists' recommendations in the North Carolina Medicaid medication therapy management program. J Am Pharm Assoc. 2010;50(3):347-53.

12. Sutton RT, Pincock D, Baumgart DC, et al. An overview of clinical decision support systems: benefits, risks, and strategies for success. NPJ Digit Med. 2020;3:17. 